\title{
Primary Alveolar Hypoventilation Syndrome Complicated with Antiphospholipid Syndrome
}

\author{
Johsuke HARA, Masaki FuJIMURA, Shigeharu MYOU, Yoshitaka ORIBE, \\ Shiho FURUSHO, Masahide YAMAZAKI and Shinji NAKAO
}

\begin{abstract}
A 32-year-old woman was transported to our hospital by ambulance because of loss of consciousness and breathing induced by drug intoxication. After general status was recovered, her arterial blood gas analysis under breathing room air revealed hypercapnia and hypoxemia which were caused by hypoventilation. After exclusion of apparent pulmonary, neuromuscular and central nerve diseases, she was diagnosed with primary alveolar hypoventilation syndrome. She had the complication of antiphospholipid syndrome (APS), suggesting the possibility of small lesions of the brainstem due to APS, which were too small to be detected on CT or MRI; these small lesions could cause injuries to the respiratory center.
\end{abstract}

(Internal Medicine 44: 987-989, 2005)

Key words: primary alveolar hypoventilation syndrome, antiphospholipid syndrome, non-invasive positive pressure ventilation

\section{Introduction}

Primary alveolar hypoventilation syndrome (PAHS) is a rare disorder showing hypercapnia and hypoxemia without apparent associated pulmonary, neuromuscular and central nerve diseases. Disorder of the chemical respiratory control system is considered to be a cause of PAHS. We describe a case of PAHS complicated with antiphospholipid syndrome.

\section{Case Report}

A non-obese 32-year-old woman was transported to our hospital by ambulance, because of loss of consciousness and breathing after phleboclysis of diazepam and pentazocine at a family doctor on where she received medication for a headache and malaise. Her medical history was temporomandibular arthrosis and hemorrhoids. She had no history of pregnancy and was a heavy smoker.

Clinical examination revealed a temperature of $36.0^{\circ} \mathrm{C}$, consciousness level JCS-200, BP $127 / 80 \mathrm{mmHg}$, heart rate $71 \mathrm{~min}^{-1}$, respiratory rate $5 \mathrm{~min}^{-1}$. Ausculation of the chest was normal. There were no abnormalities in her skin and joints. Neurological examination was also normal respectively. Arterial blood gas analysis (BGA) under $10 \mathrm{l} / \mathrm{min}$ $\mathrm{O}_{2}$ inhalation showed $\mathrm{pH}$ 7.110, $\mathrm{PaO}_{2}$ 516.5 Torr, $\mathrm{PaCO}_{2}$ 105.7 Torr, $\mathrm{HCO}_{3}^{-} 32.8 \mathrm{mmol} / l$. The hemoglobin concentration was $15.7 \mathrm{~g} / \mathrm{dl}$, platelets were $11.4 \times 10^{4} / \mu \mathrm{l}$ and activated partial thromboplastin time was 51.3 seconds (normal range: 31.8-45.4 seconds). Blood chemistry and thyroid function were normal. Repeat laboratory studies showed positive lupus anticoagulant indicated by kaolin clotting time mixture test showing inhibitor pattern and positive $\beta 2$-glycoprotein 1 independent anticardiolipin antibody (1st $2.3 \mathrm{unit} / \mathrm{ml}$, 2nd $1.8 \mathrm{unit} / \mathrm{ml}$, normal range $<1.8 \mathrm{unit} / \mathrm{ml}$ ). Tests for antibodies to DNA, Sm, RNP, SS-A, SS-B, Scl-70 and antinuclear antibodies were negative. The chest radiograph and brain CT were normal. There was no abnormality on electrocardiogram, echocardiogram and cerebrospinal fluid examination. But sinus bradycardia was found on Holter recording (Table $1)$.

She was diagnosed with drug intoxication. She was intubated and mechanically ventilated. Her general status was recovered and she was extubated, whereas her BGA on room air showed a pH 7.366, $\mathrm{PaO}_{2} 59.3$ Torr, $\mathrm{PaCO}_{2} 59.9$ Torr, $\mathrm{HCO}_{3}^{-} 33.4 \mathrm{mmol} / \mathrm{l}, \mathrm{A}-\mathrm{aDO}_{2} 5.8$ Torr. Normal value of A$\mathrm{aDO}_{2}$ indicated hypoventilation state. The pulmonary function tests were normal (\% VC 96.1\%, \% $\mathrm{FEV}_{1} 83.1 \%$, $\mathrm{FEV}_{1} / \mathrm{FVC}$ 87.1\%, \%DLco 96.6\%, \%DLco/VA 107.0\%). The response to $\mathrm{CO}_{2}$ was assessed by the rebreathing method. The response of respiratory minute volume $\left(\Delta \mathrm{V}_{\mathrm{E}} /\right.$

From the Respiratory Medicine, Cellular Transplantation Biology, Kanazawa Graduate University School of Medicine, Kanazawa

Received for publication January 11, 2005; Accepted for publication May 18, 2005

Reprint requests should be addressed to Dr. Hiroyuki Yamashita, the First Department of Internal Medicine, Kyushu University, 3-1-1 Maidashi, Higashiku, Fukuoka 814-0133 
Table 1. Polysomnography and Holter Recordings

\begin{tabular}{ll}
\hline Polysomnography & \\
\hline Total sleep time & $231.5 \mathrm{~min}$ \\
\%NREM sleep & $96.3 \%$ \\
\%REM sleep & $3.7 \%$ \\
Apnea index & 0 \\
Hypopnea index & 0.3 \\
Lowest $\mathrm{SpO}_{2}$ & $59 \%$ \\
Mean $\mathrm{SpO}_{2}$ & $86 \%$ \\
\hline & \\
\hline Holter recording & \\
\hline Minimum heart rate & 33 b.p.m. \\
Maximum heart rate & 79 b.p.m. \\
Average heart rate & 56 b.p.m. \\
\hline
\end{tabular}

NREM: non rapid eye movement, REM: rapid eye movement.

$\left.\triangle \mathrm{PET}_{\mathrm{CO} 2}\right)$ was 0.259 , indicating the impairment of the respiratory response to hypercapnia. The hyperventilation test improved the arterial blood gas abnormalities (from $\mathrm{PaO}_{2}$ 68.8 Torr, $\mathrm{PaCO}_{2}$ 58.4 Torr to $\mathrm{PaO}_{2} 111.0$ Torr, $\mathrm{PaCO}_{2} 36.4$ Torr, without $\mathrm{O}_{2}$ inhalation). Overnight polysomnography showed marked oxygen desaturation (59\% at minimum) due to hypoventilation. Apparent apnea was not observed (Table 1). MRI of the brain stem, electromyography, nerve conduction velocity and muscle CT were normal. The patient was diagnosed with PAHS conclusively. She was also diagnosed to have primary APS, because brain MRI showed an old ischemic change in the left parietal lobe (Fig. 1) and there was no clinical sign or laboratory data that suggested autoimmune disease and collagen vascular disease, including systemic lupus erythematosus. And Tc-99m ECD (ethyl cysteinate dimmer) brain single photon emission computed tomography revealed focal hypoperfusion brain lesion in the left frontal and left temporal lobe.

Bilevel continuous positive airway pressure (BiPAP) at night was induced and thereafter her headache, malaise and arterial blood gas abnormalities in the daytime were improved. We started treatment with aspirin for APS. But, she became less talkative, lethargic, seclusive with a sad look. Mental state examination revealed psychomotor retardation, depressed mood, depressive ideation and she was diagnosed as a case of major depression fulfilling DSM-IV criteria by a psychiatrist of our hospital.

\section{Discussion}

PAHS is a rare condition characterized by a marked depression of respiratory drive during sleep. This disorder is believed to result from failure of chemical respiratory control system of the brain stem neuronal networks. Recently, the criteria for the diagnosis of PAHS have been recommended

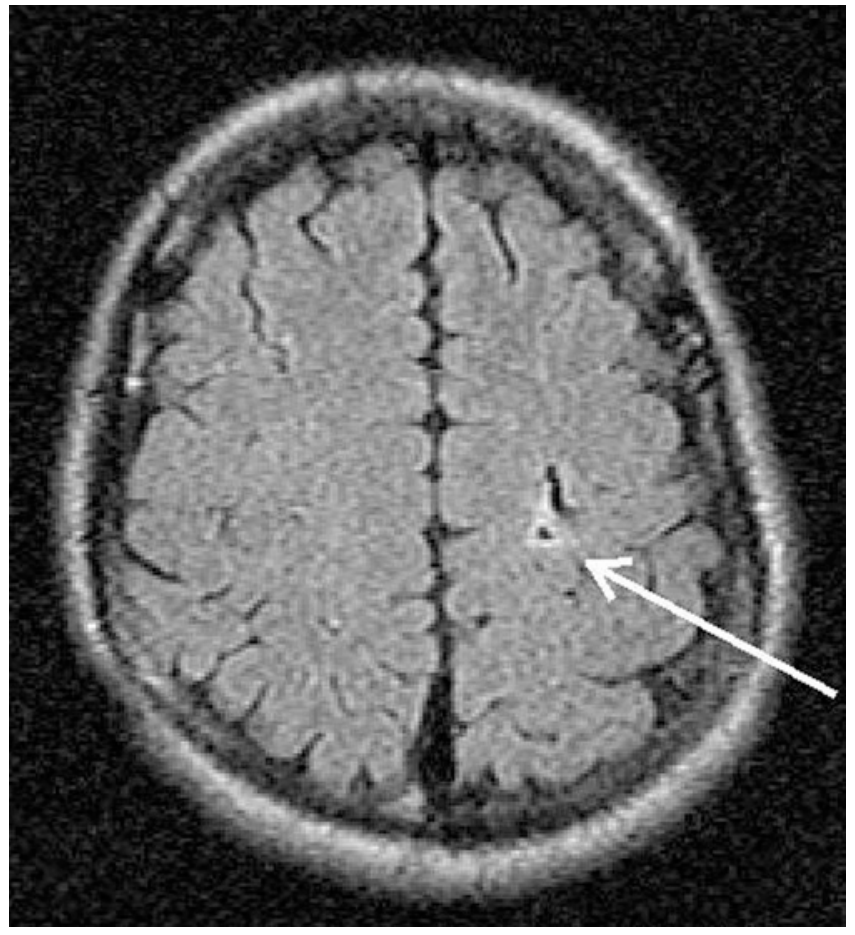

Figure 1. A FLAIR axial T2-weighted MRI of the cervical showed an old ischemic change in the left parietal lobe (arrow).

by the Respiratory Failure Research Committee of the Japanese Ministry of Health and Welfare (1) (Table 2). The present case satisfied the criteria.

Cases of central hypoventilation syndrome associated with brainstem infarction have been reported previously (2, 3). The present patient had no risk factor for cerebrovascular disease, excluding smoking. She was also diagnosed with APS fulfilling the Sapporo criteria for APS (4). APS often causes cerebral infarction. The lesion of the brain stem is difficult to detect because the lesion is often small and the brain stem is adjoined to bones of the skull base inducing susceptibility (5). In the present case, MRI on brainstem showed no abnormal findings, but the small lesions of the brainstem due to APS might have caused injuries to the respiratory center.

She was also diagnosed as major depression. APS can cause psychiatric disorders, such as depression and psychosis associated with antiphospholipid antibodies (aPL) (6). The presumed pathophysiologic mechanism underlying these manifestations is thought to be a result of cerebral ischemia in some, but not in all cases. And it is suggested that some of the aPL-associated neurological manifestations may be due to an aPL-brain phospholipid interaction, but not to a thrombotic mechanism (7). In the present case, the brain stem lesion involving the respiratory center might not be thrombotic, and thus, MRI of the brainstem showed no abnormal findings.

PAHS occurring during infancy is called congenital central alveolar hypoventilation syndrome (CCHS). It has been 
Table 2. Criteria for Diagnosis Primary Hypoventilation Syndrome

1) Chronic hypercapnia $\left(\mathrm{PaCO}_{2} \geqq 45\right.$ Torr $)$

2) Exacerbation of hypoxemia during sleep (desaturation over $4 \%$ in $\mathrm{SpO}_{2}$, over 5 minutes of $\mathrm{SpO}_{2}<90 \%$, or $\mathrm{SpO}_{2} 85 \%$ )

3) Improvement of hypercapnia after voluntary hyperventilation ( 5 Torr decrease in $\mathrm{PaCO}_{2}$ )

4) Almost normal pulmonary function $(\% \mathrm{VC}>60 \%$ and $\mathrm{FEV} 1 \%>60 \%)$. Excluding organic pulmonary disease.

5) Exclusion of dysfunction of the respiratory center or respiratory muscle paralysis due to medication or neuromuscular diseases.

6) No organic disease of the central nervous system based on the neurolophysiological examination and morphological examination.

7) Body mass index $<30 \mathrm{~kg} / \mathrm{m}^{2}$

8) Exclusion of typical sleep apnea syndrome.

A person who satisfies all of the above criteria is diagnosed as primary alveolar hypoventilation syndrome.

reported that $16 \%$ of CCHS occurs with Hirschsprung's disease (8). Although the relationship between CCHS and adult onset PAHS is unclear, the familial occurrence of PAHS may suggest an underlying genetic mechanism (9). Funada et al (10) reported a PAHS patient with decreased movement and marked dilatation of the colon suggestive of Hirschsprung's disease. They also represented that some adult PAHS may be a mild type of CCHS (10) Patients with CCHS often manifest improper autonomic nervous system including heart rate control. Sinus bradycardia has been identified in CCHS patients (11). In the present case, a marked decrease in the heart rate was observed on Holter recordings.

Recently non-invasive positive pressure ventilation (NIPPV) has become available for PAHS. NIPPV therapy appears to be useful because it is non-invasive, the equipment is simpler, its costs are lower and there is no risk of airway obstruction $(12,13)$. In the present case, after application of NIPPV therapy only during the nighttime, daytime arterial blood gas abnormalities improved and clinical complaints were resolved. Similar cases have been previously reported $(14,15)$. The improvement in ventilation not only at night but also in the daytime may be the result of improved central respiratory drive, improved chemoreceptor function, removal of hypoxic ventilatory depression and rest of the respiratory muscle.

In conclusion, we reported a case of PAHS. To our knowledge, this is the first report of PAHS complicated with APS. APS might have caused injuries to the respiratory center. NIPPV therapy improved arterial blood gas abnormalities and the clinical complaints.

Acknowledgements: We thank Professor Yoshifumi Koshino and his group of the Department of Psychiatry and Neurobiology, Kanazawa University Graduate School of Medical Science for polysomnography. We also appreciate Professor Masahito Yamada and his group of the department of Neurology and Neurobiology of Aging, Kanazawa University Graduate School of Medical Science for their technical assistance.

\section{References}

1) Kimura H, Horie Y, Okada O, et al. Criteria for diagnosis and severity, and prognosis of primary alveolar hypoventilation and obesity hypoventilation syndrome. 1999 study reports of the Respiratory Failure Research Committee of Japanese Ministry of Health and Welfare, 1999: 83-86.

2) Bogousslavsky J, Khurana R, Deruaz JP, et al. Respiratory failure and unilateral caudal brainstem infarction. Ann Neurol 28: 668-673, 1990.

3) Schafer D, Bianchi O, Greulich W, Schafer C, Schafer T, Schlafke ME. Sleep and breathing disorders in patients with brain stem lesions. Wien Med Wochenschr 146: 296-298, 1996 (in German, Abstract in English).

4) Wilson WA, Gharavi AE, Koike T, et al. International consensus statement on preliminary classification criteria for definite antiphospholipid syndrome: report of an international workshop. Arthritis Rheum 42: 1309-1311, 1999.

5) Etgen T, Grafin von Einsiedel H, Rottinger M, Winbeck K, Conrad B, Sander D. Detection of acute brainstem infarction by using DWI/MRI. Eur Neurol 52: 145-150, 2004.

6) Sanna G, Bertolaccini ML, Cuadrado MJ, Khamashta MA, Hughes GR. Central nervous system involvement in the antiphospholipid (Hughes) syndrome. Rheumatology (Oxford) 42: 200-213, 2003.

7) Brey RL, Escalante A. Neurological manifestions of antiphospholipid antibody syndrome. Lupus 7 (suppl 2): S67-74, 1998.

8) Weese-Mayer DE, Silvestri JM, Marazita ML, Hoo JJ. Congenital central hypoventilation syndrome. Inheritance and relation to sudden infant death syndrome. Am J Med Genet 47: 360-367, 1993.

9) Hida W. Novel aspects of primary alveolar hypoventilation. Intern Med 40: 463-464, 2001.

10) Funada Y, Nishimura Y, Kamemura K, et al. Familial adult onset primary alveolar hypoventilation syndrome. Intern Med 40: 526-531, 2001.

11) Weese-Mayer DE, Silvestri JM, Volgman AS, Jones P, Barnes SD. Congenital central hypoventilation syndrome (CCHS). Rhythm disturbance before, during, and after bronchoscopy (abstract). Am J Respir Crit Care Med 151: A618, 1995.

12) Idiopathic congenital central hypoventilation syndrome: diagnosis and management. American Thoracic Society. Am J Respir Crit Care Med 160: 368-373, 1999.

13) Krachman S, Criner GJ. Hypoventilation syndromes. Clin Chest Med 19: 139-155, 1998.

14) Guilleminault C, Stoohs R, Schneider H, Podszus TJ, Peter H, vonWichert P. Central alveolar hypoventilation and sleep. Treatment by intermittent positive pressure ventilation through nasal mask in an adult. Chest 96: 1210-1212, 1989.

15) Miyake $Y$, Iwanaga $T$, Kishikawa R, et al. A case of primary hypoventilation syndrome improved by BiPAP. Kokyuu 16: 1597-1601, 1997 (in Japanese, Abstract in English). 